\title{
Emended Descriptions of the Genus Alcaligenes and of Alcaligenes faecalis and Proposal That the Generic Name Achromobacter be Rejected: Status of the Named Species of Alcaligenes and Achromobacter
}

\author{
Request for an Opinion \\ MARGARET S. HENDRIE, A. J. HOLDING, and J. M. SHEWAN
}

Torry Research Station, Aberdeen, Scotland, United Kingdom, and Department of Microbiology, University of Edinburgh, Scotland, United Kingdom

\begin{abstract}
The descriptions of the genus Alcaligenes Castellani and Chalmers and Alcaligenes faecalis Castellani and Chalmers have been emended. It is requested that the Judicial Commission issue an Opinion rejecting the name Achromobacter Bergey et al. as a nomen dubium. It is proposed, on the basis of a comparison of type and reference strains, that Alcaligenes denitrificans Leifson and Hugh, Achromobacter arsenoxydans-tres Turner, and Alcaligenes odorans (Málek and Kazdová-Kožišková) Málek et al. be considered as subjective synonyms of $A$. faecalis. The proposed taxonomic status of all known previously described species of the genera Alcaligenes and Achromobacter is indicated in appendixes.
\end{abstract}

Emendation of description of the genus Alcaligenes Castellani and Chalmers. Extensive studies on the morphology and physiology of the gram-negative asporogenous rods have been undertaken in recent years by Baumann et al. (10, 11). De Ley et al. (56-58), Cabassi et al. (38), Samuels et al. (131), and De Ley and Shewan (unpublished data). These studies have revealed that strictly aerobic. peritrichous bacteria (with the exception oi those also possessing anaerobic respiratory mechanisms) should be considered for inclusion in the genus Alcaligenes. No valid criteria that justify the separation of Achromobacter from Alcaligenes have been established. Organisms such as those belonging to Acetobacter, Agrobacterium, or Rhizobium possess these characteristics, but they also possess special physiological or ecological properties that exclude them from Alcaligenes and Achromobacter. These two prime characteristics, therefore, exclude from Alcaligenes and Achromobacter bacteria that are facultatively anaerobic, nonmotile, or polarly flagellated. These bacteria are allocated to the family Enterobacteriaceae, the Acinetobacter-Moraxella group, and the family Pseudomonadaceae, respectively.

As a result of these previous studies and of unpublished investigations on 300 extant strains of 270 named species previously classified as belonging to either Alcaligenes or Achromobacter, the following emended description of the genus Alcaligenes is proposed.
Rods or coccoid cells, 0.5 to 0.6 by 1.2 to 2.6 $\mu \mathrm{m}$, usually occurring singly. Motile by means of one to four (occasionally up to eight) flagella; the flagella may be degenerate. Cells are peritrichous. No resting stages are known. Gram negative.

Chemoorganotrophic; some species are chemolithotrophic with $\mathrm{H}_{2}$; metabolism is respiratory, never fermentative. Molecular oxygen is the final electron acceptor.

Strictly aerobic except for some strains that are capable of anaerobic respiration in the presence of nitrate or nitrite, which act as alternate electron acceptors.

Most strains (except those of $A$. aquamarinus) have simple nitrogenous nutritional requirements and produce very turbid growth in liquid media with ammonium or nitrate salts as the sole nitrogen source; some strains require organic nitrogen compounds (amino acids and/or vitamins). Gaseous nitrogen is not fixed.

Not actively proteolytic in casein or gelatin media. Cellulose, chitin, and agar are not hydrolyzed. Oxidase is produced (Kovacs test).

Optimal temperatures are between 20 and $37 \mathrm{C}$. All species grow quickly at $\mathrm{pH} 7.0$.

The guanine plus cytosine $(\mathrm{G}+\mathrm{C})$ content of the deoxyribonucleic acid ranges from 58 to 70 $\mathrm{mol} \%$.

Attempts by many workers to isolate a strain that possesses the described characteristics of the type species of Achromobacter, A. liquefaciens (Eisenberg) Bergey et al., have failed. This lack of 
a neotype strain has prevented more detailed investigations of the genus from being carried out.

Proposed rejection of the generic name Achro mobacter Bergey et al. The genus named Achromobacter was first instituted in 1923 by the Committee of the Society of American Bacteriologists (16) to accommodate nonpathogenic, small to medium-sized, motile or nonmotile rods that were aerobic, nonpigmented, and asporogenous and which occurred principally in soil and water. Such rods had been described previously by Eisenberg (61), Lehmann and Neumann (100), Mez (110), Migula (111), Chester (44), and Ford (67) under a variety of names, including Bacillus liquefaciens and Bacillus liquidus. It was recognized early (51), however, that "there seems to be no recognisable distinction between Alcaligenes and similar organisms which were (then) placed in Achromobacter" and that Achromobacter is an ill-defined genus with a possibly unrecognizable type species.

In mosi of the early work, little or no mention is made of the type of flagellation of organisms placed in Achromobacter, and only in Bergey's Manual (16), when the genus Achromobacter was first named, were other additional characteristics, including flagella position, added. A. liquefaciens was listed in Bergey's Manual (16) as the type species and was described as possessing peritrichous cells; $A$. liquidum was described as possessing polar flagella. Documentation of the specific reasons for ascribing peritrichous cells to $A$. liquefaciens is lacking.

As described in Bergey's Manual (16), organisms of the genus Alcaligenes differed from those of Achromobacter in that the former were of intestinal origin and did not ferment carbohydrates. The description of Achromobacter did not mention the action on carbohydrates but stated that the organisms were from soil and water. Both genera included nonmotile and motile forms, although no polarly flagellated cells were described in Alcaligenes. Clearly, even in this early publication, grounds for separation of the genera were questionable. Succeeding editions of Bergey's Manual emphasized the action on carbohydrates and, for Alcaligenes, in the 7 th edition it was specifically stated that carbohydrates were not attacked. The action of Achromobacter liquefaciens on carbohydrates has not been reported. As a result of the description presented in Bergey's Manual (16), the genus Achromobacter was used to place a very heterogeneous collection of organisms reputed to be aerobic, gram-negative, motile or nonmotile bacteria occurring in soils, water, foods, etc.

The most recent descriptions of the genera Alcaligenes (25) and Achromobacter (26) are as follows.
Alcaligenes Castellani and Chalmers 1919 (41): "Rods which are either motile by means of peritrichous flagella or non-motile. Gram-negative. May or may not liquefy gelatin and solidified blood serum. Litmus milk turned alkaline, with or without peptonization. Carbohydrates not utilized. Acetylmethylcarbinol not produced. Chromogenesis, when it occurs, is grayish-yellow, brownish-yellow or yellow. Generally found in the intestinal tracts of vertebrates or in dairy products. The type species is Alcaligenes faecalis Castellani and Chalmers."

Achromobacter Bergey et al. 1923 (16): "nonpigment-forming (at least no pigment formed on agar or gelatin) rods. Motile by means of peritrichous flagella or non-motile. Gram-negative. Litmus milk faintly acid to unchanged or alkaline. Occur in salt- to fresh-water and in soil. The type species is Achromobacter liquefaciens (Eisenberg) Bergey et al."

Although there exists a reference strain of Alcaligenes faecalis that accords with the original description of Castellani and Chalmers, there is no extant culture of Achromobacter liquefaciens, and the most recent attempt (149) to establish a neotype failed because all extant cultures did not comply with the type description and, in fact, were found to be gram-positive rods. Moreover, both the original and subsequent descriptions of Achromobacter liquefaciens were so indeterminate and apparently were not based on the examination of extant cultures that they attracted a heterogeneous collection of what are now obviously widely divergent strains. On these grounds, the Judicial Commission is asked to issue an Opinion rejecting the generic name Achromobacter as a nomen dubium.

Emended description of Alcaligenes faecalis Castellani and Chalmers (1919) (41). The following revised description of Alcaligenes faecalis is based upon an investigation of available strains, including the reference strain ATCC $8750(=$ NCIB 8156).

Coccoid rods, 0.5 to 0.6 by $2.0 \mu \mathrm{m}$, usually occurring singly. Motile by means of one to eight flagella. Cells are peritrichous. Gram negative. No special pigments are produced. A characteristic strawberry-like odor is produced by some strains.

Acetate, propionate, butyrate, and other organic acids are utilized as sole carbon and energy sources, as are aspartic acid, asparagine, histidine, glutathione, and some other organic nitrogenous compounds. The utilization of carbohydrates and chemolithotrophic growth using $\mathrm{H}_{2}$ gas have not been demonstrated. Oxidation of arsenite, which is very active in some strains, does not support chemolithotrophic growth.

Sole nitrogen sources include ammonium and 
nitrate salts, some amino acids, and other organic nitrogenous compounds. Some strains are able to denitrify by respiring anaerobically in the presence of nitrate or nitrite to produce nitrogen gas. This ability may be lost on prolonged aerobic cultivation. Litmus milk is turned alkaline. The optimal temperature is between 25 and $37 \mathrm{C}$. The guanine plus cytosine content of the deoxyribonucleic acid is $58.9 \mathrm{~mol} \%$.

A comparison of the properties, confirmed by us, of the type strains of three previously described species of Alcaligenes, viz. Alcaligenes denitrificans Leifson and Hugh 1954 (101) (strain NCTC 8582 [ = ATCC $15173=$ CIP 6081]), Achromobacter arsenoxydans-tres Turner 1954 (151) (strain
NCIB 8687), and Alcaligenes ndorans Málek and Kazdová-Kožišková 1946, 189 (106), Málek, Radochova and Lysenko 1963 (107) (strain CCEB 554 [ = ATCC 15554]) (Tablel), shows that they are so similar to the reference strain of Alcaligenes faecalis (NCIB $8156=$ ATCC 8750) that their names can be regarded as subjective synonyms of Alcaligenes faecalis.

The only characteristic differentiating strains of Alcaligenes denitrificans from those of Alcaligenes faecalis is anaerobic respiration in the presence of nitrate, a property which may be lost on subculturing, and one which, in our opinion, should not be used by itself to differentiate species.

Achromobacter arsenoxydans-tres (not validly

TABLE 1. Properties possessed in common by Alcaligenes faecalis (NCIB $8156=$ ATCC 8750), Alcaligenes denitrificans (NCTC $8582=$ ATCC 15173), Alcaligenes odorans $(C C E B 554=A T C C$ 15554), and Alcaligenes arsenoxydans (NCIB 8687) as published (Castellani and Chalmers [4I], Leifson and Hugh [10I], Malek and Kazdova-Koziskova [106], and Turner [15l]) and confirmed by us

\begin{tabular}{|c|c|}
\hline Determination & Result \\
\hline Morphology & $\begin{array}{l}\text { Gram-negative non-sporeforming, coccal rods measuring } 0.5-1.0 \times 0.4-2.0 \mu \mathrm{m} \text {; } \\
\text { occurring singly, in pairs, and irregularly or in short chains; peritrichous }\end{array}$ \\
\hline \multicolumn{2}{|l|}{ Growth } \\
\hline On agar plate & Semitransparent to opaque, entire, smooth, flat, non-pigmented colonies \\
\hline In broth & Uniformly turbid; thin to moderate pellicle; strong aerobic growth \\
\hline Temperature & Mesophilic, range is from 5 to $37 \mathrm{C}$ \\
\hline Gelatin liquefaction & Negative \\
\hline Starch hydrolysis & Negative \\
\hline $\begin{array}{l}\text { Utilization of the usual } \\
\text { pentoses or hexoses }\end{array}$ & Negative \\
\hline Litmus milk & Alkaline; no other change \\
\hline $\mathrm{H}_{2} \mathrm{~S}$ production & Usually negative \\
\hline $\mathrm{NO}_{3}$ & May or may not be reduced \\
\hline Indole production & Negative \\
\hline Oxidase production & Positive \\
\hline Arsenite oxidation & Slightly to strongly positive (in strains of $A$. arsenoxydans). \\
\hline Lipolysis & Variable \\
\hline Guanine + cytosine & $\begin{array}{l}56.6 \text { to } 59.4 \mathrm{~mol} \% \\
\text { Alcaligenes faecalis, } 58.9 \mathrm{~mol} \% \\
\text { Alcaligenes odorans, } 56.6 \mathrm{~mol} \% \\
\text { Alcaligenes arsenoxydans, } 59.4 \mathrm{~mol} \%\end{array}$ \\
\hline Nutrition & $\begin{array}{l}\text { No vitamin requirements (i.e., can grow in Hutner mineral salt solution plus (per } \\
\text { liter): glucose }(5 \mathrm{~g}) \text {, sodium nitrate }(1 \mathrm{~g}) \text {, sodium acetate }(1 \mathrm{~g}) \text {, sodium succinate } \\
\left.(1 \mathrm{~g}) \text {, calcium gluconate }(1 \mathrm{~g}) \text {, and } \mathrm{KNO}_{3}(1 \mathrm{~g}) \text {, (see ref. } 85\right)\end{array}$ \\
\hline
\end{tabular}


published because it is not a binary combination [Rule 14a(1)]) was a name proposed for strains possessing the ability to oxidize arsenite actively. However, in our hands some strains of Alcaligenes faecalis, Alcaligenes odorans CCEB 554, and Alcaligenes denitrificans CIP 6081 oxidize arsenite, but much less actively than $A$. arsenoxydanstres.

Alcaligenes odorans is a name proposed for an organism producing a characteristic odor variably described as being similar to valerian tincture or as strawberry-like. However, odor production by the strains investigated is a variable, apparently unreliable characteristic, and in our opinion organisms possessing it do not merit specific ranking on this basis alone.

Therefore, it is proposed that Alcaligenes denitrificans, Achromobacter arsenoxydans-tres, and Alcaligenes odorans be considered as subjective synonyms of Alcaligenes faecalis.

Status of previously described species of Al- caligenes and Achromobacter. During the reappraisal of the characteristics of the genus Alcaligenes, a comprehensive list of the names of all described species of Alcaligenes and Achromobacter was prepared. Certain species are acceptable under the proposed redescription of the genus Alcaligenes (Appendix Ia), but an opportunity to investigate certain named species of $A$ chromobacter, Agrobacterium, and Pseudomonas that might be acceptable (Appendix Ib) has not been possible. Other species that either are of unknown status because there are no known extant type strains (Appendix II) or do not conform to the revised description (Appendix III) have been excluded from the genus Alcaligenes. Appendix IV lists species that were originally placed in Achromobacter and that have some properties in common with Alcaligenes but that were transferred to other genera in the 7th edition of Bergey's Manual. Appendix $\mathrm{V}$ lists species names which have not been validly published.

\section{APPENDIX Ia}

The following extant species previously placed in either the Alcaligenes or Achromobacter appear to be acceptable as species of Alcaligenes.

\begin{tabular}{|c|c|c|}
\hline Species & $\begin{array}{c}\text { Type or reference } \\
\text { strain }\end{array}$ & Reference \\
\hline $\begin{array}{l}\text { Alcaligenes faecalis } \\
\text { Achromobacter aquamarinus } \\
\text { Alcaligenes eutrophus } \\
\text { Alcaligenes paradoxus }\end{array}$ & $\begin{array}{l}\text { ATCC } 8750 \\
=\text { NCIB } 8156 \\
\text { ATCC } 14400 \\
=\text { NCMB } 557 \\
\text { ATCC } 17697 \\
\text { ATCC } 17713 \\
\text { (Biotype I) } \\
\text { ATCC } 17549 \\
\text { (Biotype II) }\end{array}$ & $\begin{array}{l}\text { Castellani and Chalmers } 1919(41) \\
\text { ZoBell and Upham } 1944(163) \\
\text { Davis et al. } 1969(54) \\
\text { Davis et al. } 1969(54)\end{array}$ \\
\hline
\end{tabular}




\section{APPENDIX Ib}

The following extant species previously described as belonging to the genus Achromobacter, Agrobacterium or Pseudomonas appear to be acceptable as species of Alcaligenes. However, a comparison with other species has not been carried out, and their status is therefore uncertain.

\begin{tabular}{|c|c|c|}
\hline Species & $\begin{array}{l}\text { Type or reference } \\
\text { strain }^{a}\end{array}$ & Reference \\
\hline \multicolumn{3}{|l|}{ Achromobacter } \\
\hline A. agile & NCIB 9986 & $\begin{array}{l}\text { From the Institute of Microbiology, Academy } \\
\text { of Sciences, Moscow }\end{array}$ \\
\hline A. cholinophagum & $\begin{array}{l}\text { ATCC } 15918 \\
=\text { NCMB } 1501\end{array}$ & Shieh $1964(134)$ \\
\hline A. pestifer & ATCC 15445 & Bergey et al. (16) \\
\hline A. starkeyi & NCIB 10688 & Ruiz-Herrera $1970(130)$ \\
\hline \multicolumn{3}{|l|}{ Agrobacterium } \\
\hline A. agile & & Ahrens $1968(3)$ \\
\hline A. aggregatum & & Ahrens $1968(3)$ \\
\hline A. ferrugineum & & Ahrens and Rheinheimer 1967 (4) \\
\hline A. gelatinovorum & & Ahrens $1968(3)$ \\
\hline A. kieliense & & Ahrens $1968(3)$ \\
\hline A.luteum & & Ahrens and Rheinheimer 1967 (4) \\
\hline A. sanguineum & & Ahrens and Rheinheimer 1967 (4) \\
\hline A. polvspheroidum & & Nikitin and Vasilyeva $1967(114)$ \\
\hline \multicolumn{3}{|l|}{ Pseudomonas } \\
\hline P. oxalaticus & & Khambata and Bhat $1953(92)$ \\
\hline
\end{tabular}

${ }^{a}$ The strains investigated at NCIB possess peritrichous cells and appear to belong to the genus Alcaligenes. 


\title{
APPENDIX 11
}

There are no known extant type strains of the following species which were placed either in the genus $A$ lcaligenes or Achromobacter. Their status is, therefore, unknown.

\author{
Alcaligenes \\ A. albus (Buchanan and Hammer 1915) Haupt 1935 (78) \\ A. alkalofoetidus Castellani and Florence 1930 (42) \\ A. denieri Corbet 1930 (53) \\ A. denitrificans (Jensen 1898) Monias 1928 (112) \\ A. faecalis var. mariense Hauduroy et al. 1937 (77) \\ A. faecalis subsp. radicans Evans 1931 (65) \\ A. hemolysans Henriksen 1936 (82) \\ A. lenis de Assis 1930 (55) \\ A. recti (Ford 1903) Bergey et al. 1923 (16) \\ A. stevensae Brown 1927 (36) \\ A. vivax (Archibald 1918) Castellani and Chalmers 1919 (41)
}

\section{Achromobacter}

A. aceris (Edson and Carpenter 1912) Bergey et al. 1934 (15)

A. album (Pagliani, Maggiora, and Fratini 1887) Bergey et al. 1923 (16)

A. alcaliaromaticum (Berlin 1927) Bergey et al. 1930 (18)

A. amylovorum (Rubentschik 1926) Bergey et al. 1930 (18)

A. centropunctatum (Jensen 1898) Bergey et al. 1923 (16)

A. cycloclastes (Gray and Thornton 1928) Bergey et al. 1930 (18)

A. dendriticum (Lustig 1893) Bergey et al. 1925 (17)

A. formosum (Ravenel 1896) Bergey et al. 1923 (16)

A. galophilus Bergey et al. 1930 (18)

A. gasoformans (Eisenberg 1891) Bergey et al. 1923 (16)

A. hyalinum (Jordan 1890) Bergey et al. 1923 (16)

A. inunctum (Pohl 1892) Bergey et al. 1923 (16)

A. iophagus (Gray and Thornton 1928) Bergey et al. 1930 (18) (NCMB 1051 [ = CCM 281] does not possess the described characters. The existence of any other strain from the original source is not known.)

A. liquefaciens (Eisenberg 1891) Bergey et al. 1923 (16) (ATCC 15716, isolated by Tulecke et al. [149] and proposed as a neotype strain, does not possess the characteristics ascribed to the species by Bergey et al. or to the strain described by Tulecke et al.)

A. litoralis (Russell 1892) Bergey et al. 1923 (16)

A. mucidus Alford and McCleskey 1943 (5)

A. nitrificans (Chester 1897) Bergey et al. 1923 (16)

$A$. raveneli (Chester 1901) Bergey et al. 1923 (16)

A. reticulare (Jordan 1890) Bergey et al. 1923 (16)

A. reticularum (Jordan 1890) Bergey et al. 1923 (16)

A. rugosum (Chester 1897) Bergey et al. 1923 (16)

A. sewerinii Bergey et al. 1923 (16)

A. solitarium (Ravenel 1896) Bergey et al. 1923 (16)

A. stolonatum (Adametz-Wichmann according to Adametz 1888) Krasil'nikov 1949 (98)

A. stoloniferum (Pohl 1892) Bergey et al. 1923 (16)

A. superficialis (Jordan 1890) Bergey et al. 1923 (16)

A. terrestris Spalla et al. 1961 (139)

A. thalassius ZoBell and Upham 1944 (163)

$A$. venosum (Vaughan 1892) Bergey et al. 1923 (16)

A. visco-symbioticum (Buchanan and Hammer 1915) Bergey et al. 1930 (18) 


\section{APPENDIX III}

The following species, previously assigned either to Alcaligenes or Achromobacter, are excluded from Alcaligenes because they do not conform to the genus $A$ lcaligenes as it is now defined. Culture collection accession numbers are quoted where extant strains are available.

Species

Present generic status and/or reason for rejection (from original description if no reference is given)

\section{Alcaligenes}

A. ammoniagenes (Cooke and Keith 1927) Bergey et al. 1930 (18) (ATCC 6871; ATCC 6872)

A. bookeri (Ford 1903) Bergey et al. 1923 (16)

A. giganteus Sonnabend 1963 (138)

A. lipolyticus (Evans 1916) Pacheco 1933 (116)

A. marshallii (Buchanan and Hammer 1915) Brisou and Prévot 1954 (34)

A. metalcaligenes (Castellani and Chalmers 1919) Brisou and Prévot 1954 (34)

A. radiobacter (Beijerinck and van Delden 1902) Conn 1939 (48) (E.X.3.24.2 = NCIB $9042=$ ATCC 19358; ATCC $6466=$ NCIB 8149 )

A. tolerans Abd-el-Malek and Gibson 1952 (1) (Gibson A3 = NCIB 8551 = ATCC 19359)

A. viscolactis (Mez 1898) Breed 1957 (25) (Syn. A. viscosum Weldin 1927) (ATCC $9036=$ NCIB 8154) Achromobacter

A. acidum (Chester 1901) Bergey et al. 1923 (16)

A. aerophilum (Rubentschik 1926) Bergey et al. 1930 (18)

A. ambiguum (Wright 1895) Bergey et al. 1923 (16)

A. ammoniagenes Wiidik 1950 (158)

A. amylolyticus Rubentschik and Goicherman 1935 (127)

A. anaerobium Shimwell 1937 (135)

A. anitratum (Schaub and Hauber 1948) Brisou 1953 (29)

A. arabinosaceum Patrick and Werkman 1933 (118)

A. arcticum Rusakova and Butkevich 1941 (128)

$A$. argenteophosphorescens (Katz 1888) Bergey et al. 1930 (18)

A. aromafaciens (Chester 1901) Bergey et al. 1923 (16)

A. arvillum (Gray and Thornton 1928) Bergey et al. 1934 (15)

A. azotogena (ZoBell and Upham 1944) Brisou 1955 (31)

A. buckleii

Gram positive; Brevibacterium (Breed 1953) (24)

NCIB 8155 (=ATCC 9128 = NCTC 6535) possessed polar flagella (NCIB, unpublished data). Letter from R. Hugh (1959) to NCIB stated that this strain was no longer distributed as an Alcaligenes sp. by ATCC and NCTC. It is no longer listed in the ATCC or NCIB catalogues. The existence of other strains is not known.

Nonmotile, facultatively anaerobic, oxidase negative

Gram positive (Evans 1918) (64)

Nonmotile

Nonmotile

Agrobacterium (Conn 1942) (49)

Nonmotile

Nonmotile; Acinetobacter (Baumann, Doudoroff and Stainer 1968) (10, 11)

Nonmotile

Gram positive

Terminal flagellum; Pseudomonas (Haynes 1957) (79)

Polar flagella

Indole produced

Zymomonas (Kluyver 1957) (96)

Nonmotile; Acinetobacter (Brisou and Prévot 1954) (34)

Nonmotile, ferments lactose, methyl red positive

Polar flagella

Luminescent

Illegitimate specific epithet (Rule 24b); synonym of Bacterium connii Migula 1900 but not $A$. connii (Chester 1901) (44) Bergey et al. 1923 (16)

Polar flagella

Polar flagella, amphitrichous

Not validly published. Personal communication from J. R. Waller (on behalf of Duerre) to Shewan (1970) states that the Achromobacter sp. (as quoted in papers by Duerre and colleagues but received labeled A. buckleii by J. De Ley and by J. M. Shewan from Duerre's laboratory) is almost certainly an atypical Proteus rettgeri.

Nonmotile; illegitimate specific epithet (Rule 24b) 
Present generic status and/or reason for rejection (from original description if no reference is given)

A. candicans (Frankland and Frankland 1889) Bergey et al. 1923 (16)

A. caseinicum Gahl 1928 (70)

A. chitinophilum (Hock 1941) Brisou 1955 (31)

A. chitinovorus (Benecke 1905) Brisou 1955 (31)

A. citroalcaligenes Mannheim and Stenzel 1962 (108) (NCTC 10307 = ATCC 17908; type strain)

A. citrophilum (Rubentschik 1926) Bergey et al. 1930 (18)

A. coadunatum (Wright 1895) Bergey et al. 1923 (16)

A. coccoideum (Chester 1901) Bergey et al. 1923 (16)

A. conjunctivae Mannheim and Stenzel 1962 (108)

(NCTC $10304=$ ATCC 17905; type strain)

A. connii (Chester 1901) Bergey et al. 1923 (16)

A. cruciviae (Gray and Thornton 1928) Bergey et al. 1930 (18) (NCIB $9432=$ NRRL B-1021 = NCTC 2580)

A. cyaneophosphorescens (Katz 1888) Bergey et al. $1930(18)$

A. cystinovorum Barber and Burrows 1936 (9)

A. dacunhae (Gray and Thornton 1928) Bergey et al. 1930 (18)

A. delicatulum (Jordan 1890) Bergey et al. 1923 (16)

A. delicatulus (Jordan 1890) Bergey et al. 1923 (16)

A. delmarvae Smart 1932 (136)

A. desmolyticum (Gray and Thornton 1928) Bergey et al. 1930 (18) (NCIB $9427=$ NRRL B-979 = NCTC 2578)

A. dianthi Patrick and Werkman 1933 (118)

A. echinodermis Brisou 1954 (30)

A. epsteinii Peshkov 1937 (119) (NCMB 1972 from INMI, Moscow)

A. eurydice (White 1912) Bergey et al. 1925 (17)

A. fairmountensis (Wright 1895) Bergey et al. 1923 (16)

A. fermentationis (Chester 1901) Bergey et al. 1923 (16)

A. filifaciens (Jensen 1898) Bergey et al. 1923 (16)

A. fischeri (Beijerinck 1889) Bergey et al. 1923 (16)

(ATCC $7744=$ NCMB 1281)

A. formicum (Omelianski 1903) Bergey et al. 1923 (16)

A. geminum (Chester 1901) Brisou and Prévot 1954 (34)

A. geniculatum (Wright 1895) Bergey et al. 1923 (16)

(NCIB $9428=$ CCEB $338=$ ATCC 19374)

A. georgiopolitanum Colwell et al. 1968 (45) (ATCC 23203)

A. globiformis (Conn 1928) Bergey et al. 1930 (18)

(ATCC $8010=$ NCIB 8907$)$

A. granii (Lundestad 1928) Bergey et al. 1930 (18)
Nonmotile

Polar flagellum

Chitinoclastic

Beneckea (Campbell 1957) (39)

Nonmotile; Acinetobacter (Baumann, Doudoroff, and Stanier 1968) $(10,11)$

Gram positive

Polar flagellum

Nonmotile

Nonmotile; Acinetobacter (Baumann, Doudoroff and Stanier 1968) $(10,11)$

Nonmotile

Polar flagella; Pseudomonas

Luminescent

NCIB 4854, the type strain, possesses polar flagella (unpublished results, Holding and NCIB).

Polar flagella; Pseudomonas

Synonym of $A$. delicatulus

Steinhaust (140) assigned an organism to this species but later indicated that it could be assigned to the paracolon group (Breed 1957) (26). Description of the species is insufficient to distinguish it from other groups.

Nonmotile

Polar flagella: Pseudomonas

Gram positive

Methyl red positive

The original description of this species-rods; motile with polar flagella; gram negative; actively proteolytic; produces acid and gas from carbohydrates; indole, $\mathrm{NH}_{3}$, and Voges Proskauer positive; nitrates reduced to nitrites-suggests that it would now be classified as an Aeromonas sp. This has been confirmed by the NCMB (unpublished data).

Lactobacillus (Kreig 1961) (99)

Polar flagella

Nonmotile

Nonmotile

Vibrio fischeri (Hendrie, Hodgkiss, and Shewan 1970) (80)

Escherichia (Bergey et al. 1925) (17)

Indole produced

Polar flagella

Nonmotile, deoxyribonucelic acid base ratio $41 \mathrm{~mol} \%$ guanine plus cytosine

Illegitimate specific epithet (Rule 24b); Arthrobacter (Conn and Dimmick 1947) (50)

Vibrio (Stanier 1941) (142) 
APPENDIX III-Continued

$\begin{array}{cc}\text { Species } & \begin{array}{l}\text { Present generic status and/or reason for } \\ \text { rejection (from original description if no } \\ \text { reference is given) }\end{array}\end{array}$

A. grypheae Brisou 1954 (30)

A. grypheae var. cannensis Brisou 1954 (30)

A. guttatus (Zimmerman 1890) Bergey et al. 1923 (16)

A. haemolyticus Mannheim and Stenzel 1962 (108) (NCTC 10305 = ATCC 17906; type strain)

A. haemolyticus var. alcaligenes $\mathrm{Mannheim}$ and Stenzel 1962 (108) (NCTC $10306=$ ATCC 17907; type strain)

A. haemolyticus var, glucidolytica Mannheim and Stenzel 1962 (108)

A. halestorgus (Elazari-Volcani 1940) Prévot 1961 (122)

A. halophilus Bergey et al. 1930 (18)

A. hartlebii (Jensen 1898) Bergey et al. 1923 (16)

\section{A harveyi Johnson and Shunk 1936 (88) (ATCC 14126} $=$ NCMB 1280)

A. healii (Buchanan and Hammer 1915) Bergey et al. 1925 (17)

A. histamineum Kimata and Kawai 1953 (93) (NCMB 865)

A. hyperopticum Campbell and Williams 1951 (40)

A. ichthyodermis Wells and ZoBell 1934 (156) (NCMB 407)

A. immobilis Muiller 1961 (113)(ATCC 15934)

A. indoloxidans (Gray 1928) Prévot 1961 (122) (NCIB $2760=$ ATCC 13751; NCIB $9153=$ CCM 283)

A. labrum Campbell and Williams 1951 (40)

A. lacticum Bergey et al. 1923 (16) (NCIB $8209=$ NRRL B-552; ATCC $23220=$ NCIB $8208=$ NRRL B-551)

A. larvae (Stutzer and Wsorow 1927) Bergey et al. 1930 (18)

A. lipidis (Anderson 1937) Allison et al. 1938 (6)

A. lipolyticum (Huss 1908) Bergey et al. 1925 (17)

A. lipophagum Campbell and Williams 1951 (40)

A. liquidum (Frankland and Frankland 1889) Bergey et al. 1923 (16)

A. luminosum Bergey et al. 1930 (18)

A. Iwoffi var. glucidolytica (Piéchaud, Piéchaud, and Second 1951) Brisou and Morichau-Beauchant 1952 (33)

A. marinoglutinosus ZoBell and Allen 1935 (162)

A. membranoformis ZoBell and Allen 1935 (162)

A. metalcaligenes (Castellani and Chalmers 1919) Mannheim and Stenzel 1962 (108) (NCTC $10308=$ ATCC 17909; proposed neotype strain)

A. merluccius Garmendia 1947 (71)

A. middletownii (Chester 1901) Bergey et al. 1923 (16)
Polar flagella (Brisou 1955) (31)

Polar flagella (Brisou 1955) (31)

NCIB 9405 has polar flagella (NCIB and Holding, unpublished results). Existence of other strains not knowil.

Nonmotile; Acinetobacter (Baumann, Doudoroff, and Stanier 1968) $(10,11)$

Nonmotile; Acinetobacter (Baumann, Doudoroff, and Stanier 1968) $(10,11)$

Nonmotile; synonym of $A$. haemolyticus var. haemolyticus (144), which is the same as $A$. haemolyticus: Acinetobacter (Baumann, Doudoroff and Stanier 1968) $(10,11)$

Pseudomonas

Nonmotile

ATCC 365 (= NCIB 8129), the only known strain has shoulder flagella (unpublished data, Holding and NCIB), resembling Agrobacterium. Taxonomic studies have also shown that this organism resembles Agrobacterium (148) (Bain, Houston, and Shewan, unpublished data, 1966-67) (28)

Photobacterium (Breed and Lessel 1954) (28)

Lucibacterium (Hendrie, Hodgkiss, and Shewan 1970) (80)

Nonmotile; Brevibacterium (Breed 1953) (24)

Proteus morganii (Kimata, Kawai, and Akamatsu 1958) (94)

Beneckea (Campbell 1957) (39)

Vibrio anguillarum (Hendrie, Hodgkiss, and Shewan 1971) (81)

Nonmotile

Polar flagella; Pseudomonas acidovorans (Stanier, Palleroni, and Doudoroff 1966) (143)

Beneckea (Campbell 1957) (39)

Nonmotile

Nonmotile

Nonmotile

Brevibacterium (Breed 1953) (24)

Beneckea (Campbell 1957) (39)

Polar flagella

Luminescent

Nonmotile; Moraxella (Piéchaud et al. 1951) (120)

Polar flagella; Pseudomonas (ZoBell 1943) (161)

Polar flagella; Pseudomonas (ZoBell 1943) (161)

Nonmotile; Acinetobacter (Baumann, Doudoroff, and Stanier 1968) $(10,11)$

Luminescent

Nunmotile 
APPENDIX III-Continued

Species
Present generic status and/or reason for rejection (from original description if no reference is given)
A. mirus (McBeth 1916) Prévot 1961 (122)

A. mobile Kluyver and Hoppenbrouwers 1931 (95)

(NCIB $8938=$ ATCC $10988=$ NRRL B-806)

A. mucosus (von Lingelsheim 1906) Mannheim and Stenzel 1962 (108) (NCTC $10303=$ ATCC 17904; proposed neotype strain)

A. multistriatum (Wright 1895) Bergey et al. 1923 (16)

A. nebulosum (Wright 1895) Bergey et al. 1923 (16)

A. nematophilus Poinar and Thomas 1965 (123) (ATCC 19061)

A. nenckii (Biernacki 191I) Bergey et al. 1930 (18)

A. nijibetsui Takeda 1936 (147)

A. nitrovorum (Jensen 1898) Bergey et al. 1923 (16)

A. parvulus (Conn 1922) Breed 1957 (26)

A. pastinator Goresline $1933(72)$

A. pellucidum Harrison 1929 (76)

A. perfectomarinus (ZoBell and Upham 1944) Brisou and Prévot 1954 (34)

A. perolens Turner 1927 (150) (ATCC $10757=$ NCIB 9327)

A. phosphoreum (Migula 1900) Bergey et al. 1930 (18)

A. phosphoricum (Migula 1900) Bergey et al. 1930 (18)

A. picrum Fuller and Norman 1943 (69)

A. pictorum (Gray and Thornton 1928) Bergey et al. 1930 (18) (NCIB 9152 = CCM 284)

A. pikowskyi Bergey et al. 1930 (18)

A. pinnatum (Ravenel 1896) Bergey et al. 1923 (16)

A. pinnatum var. gemminum Brisou and Prévot 1954 (34)

A. punctatum (Zimmerman 1890) Bergey et al. 1923 (16)

A. putrefaciens Derby ano Hammer 1931 (59) (ATCC $8071,8072,8073=$ NCIB 10471, 10472, 10473; cotypes)

A. radiobacter (Beijerinck and van Delden 1902) Bergey et al. 1934 (15) (E.X. $3.24 .2=$ NCIB $9042=$ ATCC 19358; ATCC $6466=$ NCIB 8149)

A. rathonis (Gray and Thornton 1928) Bergey et al. $1930(18)($ NCIB $9679=$ NRRL B-988 $=$ NCTC 2577)

A. refractans (Wright 1895) Breed 1948 (23)

A. refractum (Wright 1895) Bergey et al. 1923 (16)

A. riboflavinus (Foster 1944) Prévot 196I (122) (NCIB $8177=$ ATCC 9526)

A. rodonatum (Ravenel 1896) Bergey et al. 1923 (16)

A. salopium (Gray and Thornton 1928) Bergey et al. 1930 (18)

A. sinosum (Wright 1895) Bergey et al. 1923 (16)

A. smaragdinophosphorescens (Katz, 1888) Bergey et al. $1930(18)$

A. spermophilum Severi 1946 (133)

A. stationis ZoBell and Upham 1944 (163) (NCMB $565=$ ZoBell $622=$ ATCC 14403)

A. stearophilum (Weinzirl 1919) Bergey et al. 1923 (16)

A. stenohalis ZoBell and Upham 1944 (163)

A. stereotropis (ZoBell and Upham 1944) Brisou, Tysset, and Vacher 1959 (35)
Polar flagella

Zymomonas (Kluyver and van Niel, 1936) (97); ( $A$. mobile is not validly published, Rule 12c)

Nonmotile; Acinetobacter (Baumann, Doudoroff and Stanier 1968) $(10,11)$

Polar flagella

Polar flagella

Fermentative, oxidase negative

Nonmotile

Polar flagella

Nonmotile

ATCC 4335 has polar flagella (R. Hugh personal communication to ATCC); existence of other strains not known

Agarbacterium (Breed 1957) (27)

Polar flagella (Krasil'nikov 1949) (98)

Polar flagella

Polar flagella; forms green, water-soluble pigment; Pseudomonas (Szybalski, 1950) (146)

Luminescent

Luminescent

Nonmotile

Polar flagella; Pseudomonas

Indole produced

Indole produced

Variety of above-named species

Aeromonas (Sneiszko 1957) (137)

Pseudomonas putrefaciens (Long and Hammer 1941) (103); deoxyribonucleic acid base ratio 44 to $47 \mathrm{~mol} \%$ guanine plus cytosine (Herbert et al. 1971) (83)

Agrobacterium (Conn 1942) (49)

Polar flagella; Pseudomonas

Nonmotile

Synonym of $A$. refractans

Pseudomonas

Nonmotile

Polar flagella; Pseudomonas

Polar flagella

Luminescent

Nonmotile

Nonmotile; gram positive; Brevibacterium (Breed 1953) (24)

Gram positive

Nonmotile

Polar flagella 
APPENDIX III-Continued

\begin{tabular}{|c|c|}
\hline Species & $\begin{array}{l}\text { Present generic status and/or reason for } \\
\text { rejection (from original description if no } \\
\text { reference is given) }\end{array}$ \\
\hline $\begin{array}{l}\text { A. stutzeri (Lehmann and Neumann 1896) Bergey et } \\
\text { al. } 1930 \text { (18) }\end{array}$ & Polar flagella \\
\hline $\begin{array}{l}\text { A. stutzeri (Lehmann and Neumann 1896) Prévot } 1961 \\
\text { (122) }\end{array}$ & $\begin{array}{l}\text { Synonym of above-named species; illegitimate specific } \\
\text { epithet (Rule 24a) }\end{array}$ \\
\hline A. sulfureum Bergey et al. 1930 (18) & Gram positive \\
\hline A. tiogense (Wright 1895) Bergey et al. $1923(16)$ & Nonmotile \\
\hline $\begin{array}{l}\text { A. tralucidus (Kellerman, McBeth, Scales, and Smith } \\
\text { 1913) Prévot } 1961 \text { (122) }\end{array}$ & Polar flagella \\
\hline A. ubiquitum (Jordan 1890) Bergey et al. 1923 (16) & Nonmotile \\
\hline A. ureasophorum Campbell and Williams 1951 (40) & Beneckea (Campbell 1957) (39) \\
\hline A. viscosum Bergey et al. 1925 (17) & $\begin{array}{l}\text { Illegitimate name under Rule } 24 \mathrm{~b} \text { of the Code. Synonym } \\
\text { of Alcaligenes viscolactis. NCIB } 9408 \text {, labeled } A \text {. } \\
\text { viscosum, does not correspond to the description of } \\
\text { the species. }\end{array}$ \\
\hline A. viscosus Sreenivasan 1956 (141) (ATCC 12448) & Indole produced \\
\hline $\begin{array}{l}\text { A. winogradskii Bourlat } 1953 \text { (22) (NCIB } 9017= \\
\text { ATCC 17922) }\end{array}$ & $\begin{array}{l}\text { Not validly published as Achromobacter winogradskit } \\
\text { but listed under this name in the NCIB catalogue. } \\
\text { Nonmotile; Neisseria (Lemoigne et al. 1952) (102); } \\
\text { Acinetobacter (Brisou 1957) (32) }\end{array}$ \\
\hline A. xerosis Groupé et al. 1954 (75) & $\begin{array}{l}\text { ATCC } 14780 \text {, the type strain of } A \text {. xerosis Groupe } \\
\text { et al. is gram positive (NCIB, unpublished data). }\end{array}$ \\
\hline
\end{tabular}

\section{APPENDIX IV}

The following named species of Achromobacter (see Appendix III), which belong to other genera, are now considered to be of uncertain status and have some properties, such as being motile, peritrichate, oxidase positive, and gram negative, in common with organisms belonging to Alcaligenes.

Achromobacter chitinovorus, A. hyperopticum, A. labrum, A. lipophagum, and A. ureasophorum in Beneckea (Campbell 1957) (39)

A. pastinator in Agarbacterium (Breed 1957) (27). 


\section{APPENDIX V}

In Index Bergeyana, the following names of species of Achromobacter are considered to be not validly published.

\author{
A. alcaligenes var. alcaliaromaticum \\ A. alcaligenes var. pellucidum \\ A. alcaligenes var, ravenelli \\ $A$. alcaligenes var. rugosum \\ $A$. alcaligenes var. solitarium \\ $A$. arsenoxydans - tres \\ $A$. arvillum var. salopium \\ A. centropunctatum var. reticularei \\ A. fecaloides \\ A. formosum var. bookeri \\ $A$. formosum var. geniculatum \\ A. formosum var. nebulosum \\ A. formosum var. nitrificans \\ $A$. formosum var. stalloniferum \\ A. formosum var. superficiale \\ $A$. hyalinus var. dentriticum \\ A. hyalinus var. liquidum \\ A. litorale var. 2 \\ A. lwoffi \\ A. membrancula \\ A. miniscula \\ A. periphyta \\ A. pikowsky var. ambigua \\ A. pikowsky var. sinosum \\ $A$. rathonis var. stutzerii \\ A. recti \\ A. septicemiae \\ A. sewerini var. stutzerii
}

\section{REPRINT REQUESTS}

Address reprint requests to: Dr. J. M. Shewan, Torry Research Station, P. O. Box 131, Aberdeen AB9 8 DG, Scotland, U. K.

\section{LITERATURE CITED}

1. Abd-el-Malek, Y., and T. Gibson. 1952. Studies in the bacteriology of milk. IV. The Gram-negative rods of milk. J. Dairy Res. 19:294-301.

2. Adametz, L. 1888. Die Bacterien der Trink und Nutzwasser. Mitt öst Versuchsst Brauer. Malz Wien 1:19-63.

3. Ahrens, R. 1968. Taxonomische Untersuchungen an sternbildenden Agrobacterium-Arten aus der westlichen Ostsee. Kiel. Meersforsch. 24:147-173.

4. Ahrens, R., and G. Rheinheimer. 1967. Uber einige sternbildende Bakterien aus der Ostsee. Kiel. Meeresforsch. 23:127-136.

5. Alford, J. A., and C. S. McCleskey. 1943. A new bacterial species producing a 'musty' odor. Proc. Acad. Sci., La. 7:25-27.

6. Allison, J. B., J. A. Anderson, and W. H. Cole. 1938. The method of electrical conductivity in studies on bacterial metabolism. J. Bacteriol 36:571-586.

7. Anderson, J. A. 1937. Spoilage of cream at low temperatures, p. 19-24. Bull. Int. Ass. Milk Deal. Proc. 30th Annu. Conv.
Brisou and Prévot 1954 (34)

Brisou and Prévot 1954 (34)

Brisou and Prévot 1954 (34)

Brisou and Prévot 1954 (34)

Brisou and Prévot 1954 (34)

Turner 1954 (151)

Brisou and Prévot 1954 (34)

Brisou and Prévot 1954 (34)

Brisou and Prévot 1954 (34)

Brisou and Prévot 1954 (34)

Brisou and Prévot 1954 (34)

Brisou and Prévot 1954 (34)

Brisou and Prévot 1954 (34)

Brisou and Prévot 1954 (34)

Brisou and Prévot 1954 (34)

Brisou and Prévot 1954 (34)

Brisou and Prévot 1954 (34)

Bois and Roy, 1944 (21)

Brisou 1955 (31)

Brisou and Prévot 1954 (34)

Brisou and Prévot 1954 (34)

Brisou and Prévot 1954 (34)

Brisou and Prévot 1954 (34)

Brisou and Prévot 1954 (34)

Brisou and Prévot 1954 (34)

Brisou and Prévot 1954 (34)

Brisou and Prévot 1954 (34)

Brisou and Prévot 1954 (34)

8. Archibald, R. G. 1918. Enterica in the Sudan. J. Trop. Med. Hyg. 21:229-236.

9. Barber, H. H., and R. B. Burrows. 1936. The production of free sulphur from 1-cystine by a soil bacterium. Biochem. J. 30:599-603.

10. Baumann, P., M. Doudoroff, and R. Y. Stanier. 1968. A study of the Moraxella group I. Genus Moraxella and the Neisseria catarrhalis group. J. Bacteriol. 95:58-73.

11. Baumann, P., M Doudoroff, and R. Y Stanier. 1968. A study of the Moraxella group II Oxidasenegative species (Genus Acinetobacter) J. Bacteriol. 95:1520-1541.

12. Beijerinck, M. W. 1889. Le Photobacterium luminosum, bactérie lumineuse de la Mer du Nord. Arch. Néerl Sci. Exactes Natur. 23:401 427.

13. Beijerinck, M. W., and A. van Delden. 1902. Ueber die Assimilation des freien Stickstoffs durch Bakterien. Zentralbl. Bakteriol. Prasitenk. Infektionskr. Hyg. Abt. 2. 9:3 43.

14. Benecke, W. 1905. Über Bacillus chitinovorous, einen Chitin zersetzenden Spaltpilze. Bot. Ztg 63:227-242.

15. Bergey, D. H., R. S. Breed, B. W. Hammer, F. M. Huntoon, E. G. D. Murray, and F. C. Harrison. 1934. Bergey's manual of determinative bacteriology, 4th ed., p. 1-664. The Williams \& Wilkins Co., Baltimore.

16. Bergey, D. H., F. C. Harrison, R. S. Breed, B. W. Hammer, and F. M. Huntoon. 1923. Bergey's manual of determinative bacteriology, 1st ed., p. 
1-442. The Williams \& Wilkins Co., Baltimore.

17. Bergey, D. H., F. C. Harrison, R. S. Breed, B. W. Hammer, and F. M. Huntoon. 1925. Bergey's manual of determinative bacteriology, 2nd ed., p. 1-462. The Williams \& Wilkins Co., Baltimore.

18. Bergey, D. H., F. C. Harrison, R. S. Breed, B. W. Hammer, and F. M. Huntoon. 1930. Bergey's manual of determinative bacteriology, 3rd ed., p. 1-589. The Williams and Wilkins Co., Baltimore.

19. Berlin, A. L. 1927. Bacterium alcaliaromaticum. Rev. Microbiol. Epidemiol. 6:402-413 (p. 473-474, English summary).

20. Biernacki, W. 1911. Bacterium Nenckii Biern., ein neuer den Agar verflussigender Mikroorganismus. Zentralbl. Bakteriol. Parasitenk. Infektionskr. Hyg. Abt. 2. 29:166-169.

21. Bois, E., and G. Roy. 1944. Quelques microorganismes isolés d'intestins de morue. Natur. Can. 71:259-264.

22. Bourlat, R. 1953. Contribution a l'étude bactériologique de $B$. anitratum et des germs voisins. Leur position dans la systematique, p. 1-46. Ph.D. thesis, Université de Bordeaux. E. Drouillard, Bordeaux.

23. Breed, R. S. 1948. Genus II Achromobacter Bergey et al., p. 417-427. In R. S. Breed, E. G. D. Murray, and A. P. Hitchens (ed.), Bergey's manual of determinative bacteriology, 6th ed. The Williams \& Wilkins Co., Baltimore.

24. Breed, R. S. 1953. The families developed from Bacteriaceae Cohn with a description of the family Brevibacteriaceae Breed 1953. VI Congr. Int. Microbiol. Roma I:10-15.

25. Breed, R. S. 1957. Genus I. Alcaligenes Castellani and Chalmers 1919, p. 297-300. In R. S. Breed, E. G. D. Murray and N. R. Smith (ed.), Bergey's manual of determinative bacteriology, 7th ed. The Williams \& Wilkins Co., Baltimore.

26. Breed, R. S. 1957. Genus II. Achromobacter Bergey et al. 1923, p. 300-309. In R. S. Breed, E. G. D. Murray, and N. R. Smith (ed.), Bergey's manual of determinative bacteriology, 7 th ed. The Williams \& Wilkins Co., Baltimore.

27. Breed, R. S. 1957. Genus IV. Agarbacterium Angst, 1929, p. 322-328. In R. S. Breed, E. G. D. Murray, and N. R. Smith (ed.), Bergey's manual of determinative bacteriology, 7th ed. The Williams \& Wilkins Co., Baltimore.

28. Breed, R. S., and E. F. Lessel. 1954. The classification of luminescent bacteria. Antonie van Leeuwenhoek J. Microbiol. Serol. 20:58-64.

29. Brisou, J. 1953. Essai sur la systematique du genre Achromobacter. Ann. Inst. Pasteur (Paris) 84:812-814.

30. Brisou, J. 1954. Étude d'espèces et varietès nouvelles, d'Achromobacter isolées du milieu marin. Ann. Inst. Pasteur (Paris) 86:118-120.

31. Brisou, J. 1955. Microbiologie du Milieu Marin, p. 1-271. Flammarion, Paris.

32. Brisou, J. 1957. Contribution of l'étude de la systematique des Pseudomonadacae. Ann. Inst. Pasteur (Paris) 93:397-404.

33. Brisou, J., and R. Morichau-Beauchant. 1952. Identité biochimique entre certaines souches de $\boldsymbol{B}$. anitratum et Moraxella lwoffi. Ann. Inst. Pasteur (Paris) 82:640-643.
34. Brisou, J., and A. R. Prévot. 1954. Études de Systematique Bacterienne. X. Revision des espèces réunies dans le genre Achromobacter. Ann. Inst. Pasteur (Paris) 86:722-728.

35. Brisou, J., C. Tysset, and B. Vacher. 1959. Étude d'une souche d'Achromobacter: Achromobacter stereotropis Ann. Inst. Pasteur (Paris) 96:524-529.

36. Brown, F. M. 1927 . Descriptions of rew bacteria found in insects. Amer. Mus. Novit. 251:1-11.

37. Buchanan, R. E., and B. W. Hammer. 1915. Slimy and ropy milk. Bull. Iowa State Coll. Agr. Mech. Arts 22:209-295.

38. Cabassi, E., P. Adami, and A. Caravita. 1969. Tassonomia numerica Adansoniana di ceppi di Moraxella isolati dalla carne e dai suoi prodoti di transformazione. Arch. Vet. Ital. 20:45-63.

39. Campbell, L. L., Jr. 1957. Genus V. Beneckea Campbell, gen. nov., p. 328-332. In R. S. Breed, E. G. D. Murray and N. R. Smith (ed.), Bergey's manual of determinative bacteriology, 7 th ed. The Williams \& Wilkins Co., Baltimore.

40. Campbell, L. L., and O. B. Williams. 1951. A study of chitin-decomposing microorganisms of marine origin. J. Gen. Microbiol. 5:894-905.

41. Castellani, A., and A. J. Chalmers. 1919. Manual of tropical medicine, 3rd ed. William Wood and Co., New York.

42. Castellani, A., and M. D. Florence. 1930. Foetor oris of tonsillar origin and certain bacilli causing it. Lancet 218:623-624.

43. Chester, F. D: 1897. Report of mycologist: bacteriological work. Annu. Rep. Del. Agr. Exp. Sta. 9:38-145.

44. Chester, F. D. 1901. A manual of determinative bacteriology, p. 1-401. The Macmillan Co., New York.

45. Colwell, R. R., E. J. Smith, and G. B. Chapman. 1968. Properties of a D-quinovosamine-producing Achromobacter. Can. J. Microbiol. 14:165-171.

46. Conn, H. J. 1922. Description of an ammonia volatilizing organism found in manure. Bull. N.Y. Sta. Agr. Exp. Sta. 494:26-27.

47. Conn, H. J. 1928. A type of bacteria abundant in productive soils, but apparently lacking in certain soils of low productivity. Tech. Bull. N.Y. Sta. Agr. Exp. Sta. 138:3-26.

48. Conn, H. J. 1939. Genus III. Alcaligenes Castellani and Chalmers, p. 95-102. In D. H. Bergey, R. S. Breed, E. G. D. Murray and A. P. Hitchens (ed.), Bergey's manual of determinative bacteriology, 5th ed. The Williams \& Wilkins Co., Baltimore.

49. Conn, H. J. 1942. Validity of the genus Alcaligenes. J. Bacteriol 44:353-360.

50. Conn, H. J., and I. Dimmick. 1947. Soil bacteria similar in morphology to Mycobacterium and Corynebacterium. J. Bacteriol 54:291-303.

51. Conn, H. J., G. E. Wolfe, and M. Ford. 1940. Taxonomic relationships of Alcaligenes spp. to certain soil saprophytes and plant parasites. J. Bacteriol. 39:207-226.

52. Cooke, J. V., and H. R. Keith. 1927. A type of urea-splitting bacterium found in the human intestinal tract. J. Bacteriol. 13:315-319.

53. Corbet, A. S. 1930. An organism found in the latex of Hevea brasiliensis. J. Bacteriol. 19:321-326. 
54. Davis, D. H., M. Doudoroff, R. Y. Stanier, and M. Mandel. 1969. Proposal to reject the genus Hydrogenomonas: taxonomic implications. Int. J. Syst. Bacteriol. 19:375-390.

55. de Assis, A. 1930. Sobre Alcaligenes lenis e sua verificacao no homen. Bol. Inst. Vital. Braz. 14:1 13.

56. De Ley, J., N. Bain, and J. M. Shewan. 1967. Taxonomy of the Achromobacter and allied species. Nature (London) 214:1037-1038.

57. De Ley, J. 1968. DNA base composition and taxonomy of some Acinetobacter strains. Antonie van Leeuwenhoek J. Microbiol. Serol. 34:109-114.

58. De Ley, J., K. Kersters, J. Khan-Matsubara, and J. M. Shewan. 1970. Comparative d-gluconate metabolism and DNA base composition in Achromobacter and Alcaligenes. Antonie van Leeuwenhoek J. Microbiol. Serol. 36:193-207.

59. Derby, H. A., and B. W. Hammer. 1931. Bacteriology of butter. IV. Bacteriological studies of surface taint butter. Res. Bull. Iowa Agr. Exp. Sta. 145:387-416

60. Edson, H. A., and C. W. Carpenter. 1912. Technical description of certain bacteria in maple sap. (A) Description of Bacillus aceris (new species) Bull. Vt. Agr. Exp. Sta. 167:475-606.

61. Eisenberg, J. 1891. Bakteriologische Diagnostik, Hilfstabellen zum Gebrauche beim Praktischen Arbeiten 3 Aufl., p. 1-509. Leopold Voss, Hamburg.

62. Elazari-Volcani, B. 1940. Studies on the microflora of the Dead Sea, p. 1-119. Ph.D. thesis, Hebrew University, Jerusalem.

63. Evans, A. C. 1916. The bacteria of milk freshly drawn from normal udders. J. Infect. Dis. 18:437-476.

64. Evans, A. C. 1918. Further studies on Bacterium abortus and related bacteria. III. Bacterium abortus and related bacteria in cow's milk. J. Infect. Dis. 23:354-372.

65. Evans, A. C. 1931. A new sub-species, radicans, of Alcaligenes faecalis. Publ. Health Rep. Wash. 46:1676-1680.

66. Frankland, G. C., and P. F. Frankland. 1889 Ueber einige typische Mikroorganisms in Wasser und im Boden. Z. Hyg. Infektionskr. 6:373-400.

67. Ford, W. W. 1903. The classification and distribution of the intestinal bacteria in man. Roy. Vic. Hosp. Montreal 1(no. 5): 1 -95.

68. Foster, J. W. 1944. Microbiological aspects of riboflavin. I. Introduction. II. Bacterial oxidation of riboflavin to lumichrome. J. Bacteriol. 47:27-41.

69. Fuller, W. H., and A. G. Norman. 1943. Cellulose decomposition by aerobic mesophilic bacteria from soil. J. Bacteriol. 46:273-280.

70. Gahl, R. 1928. Description of an organism found in solutions of sodium caseinate. J. Bacteriol. $16: 35-42$

71. Garmendia, H. E. 1947. Una nueva especie de bacteria luminosa. Rev. Fac. Cienc. Quim Farm. Univ. Nac. La Plata 22:225-229.

72. Goresline, H. 1933. Studies on agar digesting bacteria. J. Bacteriol. 26:435-457.

73. Gray, P. H. H. 1928. The formation of indigotin from indole by soil bacteria. Proc. Roy. Soc. Ser. B
102:263-280.

74. Gray, P. H. H., and H. G. Thornton. 1928. Soil bacteria that decompose certain aromatic compounds. Zentralbl. Bakteriol. Parasitenk. Infektionskr. Hyg. Abt. 2 73:74-96.

75. Groupé, V., L. H. Pugh, A. S. Levin, and E. C. Herrmann. 1954. Suppression of certain viral lesions in demonstrable antiviral properties produced by Achromobacter xerosis n. sp. J. Bacteriol. 68:10-18.

76. Harrison, F. C. 1929. The discoloration of halibut. Can. J. Res. 1:214-239.

77. Hauduroy, P., G. Ehringer, A. Urbain, G. Guillot, and J. Magrou. 1937. Dictionnaire des bactéries pathogénes p. 1-597. Masson et Cie, Paris.

78. Haupt, H. 1935. Zur Systematik der Bakterien. Die für Mensch und Tier pathogenen gram negativen alkalibildenden Stäbchen-bakterien (Aerobactereae Pribram 1929 em). Ergebn. Hyg. Bakteriol. 17:175-230.

79. Haynes, W. C. 1957. Genus I. Pseudomonas Migula 1894, p. 89-152. In R. S. Breed, E. G. D. Murray and N. R. Smith (ed.), Bergey's manual of determinative bacteriology, 7th ed. The Williams \& Wilkins Co., Baltimore.

80. Hendrie, M. S., W. Hodgkiss, and J. M. Shewan. 1970. The identification, taxonomy and classification of luminous bacteria. J. Gen. Microbiol. 64:151-169.

81. Hendrie, M. S., W. Hodgkiss, and J. M. Shewan. 1971. Proposal that the species Vibrio anguillarum Bergman, 1909, Vibrio piscium David, 1927 and Vibrio ichthyodermis (Wells and ZoBell) Shewan, Hobbs and Hodgkiss, 1960 be combined as a single species, Vibrio anguillarium. Int. J. Syst. Bacteriol. 21:64-68.

82. Henriksen, S. D. 1936. Studies on the bacterial flora of the respiratory tract in acute and chronic bronchitis, bronchial asthma and lung gangrene. Avh. Nor, Vidensk. Akad. Oslo 2(no. 11):7-235.

83. Herbert, R. A., M. S. Hendrie, D. M. Gibson, and J. M. Shewan. 1971. Bacteria active in the spoilage of certain sea foods. J. Appl. Bacteriol. 34:41-50.

84. Hock, C. W. 1941. Marine chitin-decomposing bacteria. J. Mar. Res. 4:99-106.

85. Holding, A. J., and J. G. Collee. 1971. Routine biochemical tests, p. 1-32. In J. R. Norris and D. W. Ribbons (ed.), Methods in microbiology, vol. 6A. Academic Press Inc., London.

86. Huss, H. 1908. Eine feltspaltende Bakterie (Bacteridium lipolyticum) (n.sp.) Zentralbl. Bakteriol. Parasitenk. Infektionskr. Hyg. Abt. 2. 20:474 484.

87. Jensen, H. 1898. Beitrage zur Morphologie und Biologie der Denitrifikationsbakterien. Zentralbl. Bakteriol. Parsitenk. Infektionskr. Hyg. Abt. 2. 4:401-411.

88. Johnson, F. H., and I. V. Shunk. 1936. An interesting new species of luminous bacteria. $J$. Bacteriol. 31:585-592.

89. Jordan, E. O. 1890. A report on certain species observed in sewage, p. 821-824. In W. T. Sedgwick (ed.), A report on the biological work of the Lawrence Experiment Station, including an ac. count of methods employed and results obtained in the microscopical and bacteriological investigation 
of sewage and water. Report on water supply and sewerage (part II). State Board of Health of Massachusetts. Wright and Potter Printing Co., State Printers, Boston.

90. Katz, O. 1888. Preliminary remarks on phosphorescent bacteria from sea-water. Proc. Linn. Soc. N.S.W. 2:331-336.

91. Kellerman, K. F., I. G. McBeth, F. M. Scales, and N. R. Smith. 1913. Identification and classification of cellulose dissolving bacteria. Zentralbl. Bakteriol. Prasitenk. Infektionskr. Hyg. Abt. 2. 39:505-522.

92. Khambata, S. R., and J. V. Bhat. 1953. Studies on a new oxalate-decomposing bacterium, Pseudomonas oxalaticum. J. Bacteriol. 66:505-507.

93. Kimata, M., and A. Kawai. 1953. A new species of bacterium which produces large amounts of histamine on fish meats, found in spoiled fresh fish. Mem. Res. Inst. Food Sci. Kyoto Univ. 6:1-2.

94. Kimata, M., A. Kawai, and M. Akamatsu. 1958. Classification and identification of the bacteria having an activity which can produce a large amount of histamine. Mem. Res. Inst. Food Sci. Kyoto Univ. 14:33-41.

95. Kluyver, A. J., and W. J. Hoppenbrouwers. 1931. Ein merkwürdiges Gärungsbakterien: Lindner's Termobacterium mobile. Arch. Mikrobiol. 2:245-260

96. Kluyver, A. J. 1957. Genus VII. Zymomonas Kluyver and van Niel, 1936, p. 199-200. In R. S. Breed, E. G. D. Murray, and N. R. Smith (ed.), Bergey's manual of determinative bacteriology, 7th ed. The Williams \& Wilkins Co., Baltimore.

97. Kluyver, A. J., and C. B. van Niel. 1936. Prospects for a natural system of classification of bacteria. Zentralbl. Bakteriol. Parasitenk. Infektionskr. Hyg. Abt. 2. 94:369-403.

98. Krasil'nikov, N. A. 1949. Guide to the bacteria and actinomycetes, p. 1-830. Akad. Nauk. SSSR, Moscow (in Russian).

99. Kreig, A. 1961. Grundlagen der Insektenpathologie, p. 1-304. Dr. Dietrich Stein-kopff. Verlag, Darmstadt.

100. Lehmann K. B., and R. O. Neumann. 1896. Atlas und Grundriss der Bakteriologie und Lehrbuch der speciellen bacteriologischen Diagnostik. Teil II, p. 1-448. Verlag von J. F. Lehmann, Munich.

101. Leifson, E., and R. Hugh. 1954. Alcaligenes denitrificans n.sp. J. Gen. Microbiol. 11:512-513.

102. Lemoigne, H., H. Girard, and G. Jacobélli. 1952. Bactérie du sol utilisant facilement le 2-3 butanediol. Ann. Inst. Pasteur (Paris) 82:389-398.

103. Long, H. F., and B. W. Hammer. 1941. Classification of the organisms important in dairy products. III Pseudomonas putrefaciens. Res. Bull. Iowa Agr. Exp. Sta. 285:176-195.

104. Lundestad, J. 1928. Über einige ander norwegischen Kuiste isolierte Agarspaltende Arten von Meerbakterien. Zentralbl. Bakteriol. Parasitenk. Infektionskr. Hyg. Abt. 2. 75:321-344.

105. Lustig, A. 1893. Diagnostik der Bakterien des Wassers, Zweite sehr vermehrte Auflage, p. 1-128. Turm Übersetzung, Jena.
106. Malek, I., and V. Kadzová-Kožiškova. 1946. Pseudomonas odorans n.sp. novy mikrob z diagnostickeho materialu. Sb. Lék. 47(52):189-194.

107. Malek, I., M. Radochova, and O. Lysenko. 1963. Taxonomy of the species Pseudomonas odorans. J. Gen. Microbiol. 33:349-355.

108. Mannheim, W., and W. Stenzel. 1962. Zur Systematik der obligat aeroben gramnegativen $\mathrm{Di}$ plobakterien des Menschen. Zentralbl. Bakteriol. Parasitenk. Infektionskr. Hyg. Abt. I Orig. 186:55-83.

109. McBeth, I. G. 1916. Studies on the decomposition of cellulose in soils. Soil Sci. 1:437-487.

110. Mez, C. 1898. Mikroskopische Wasseranalyse, Anleitung zur Untersuschung des Wassers mit besonderer Beruicksichtigung von Trink und Abwasser, p. 1-69.

111. Migula, W. 1900. System der Bakterien, p. 1-1068. Gustav Fischer, Jena.

112. Monias, B. L. 1928. Classification of Bacterium alcaligenes, pyocyaneum and fluorescens. J. Infect. Dis. 43:330-334.

113. Muiller, G. 1961. Mikrobiologische Untersuchungen über die "Futterverpilzing durch Selbsterhitzung." III. Mitteilung Ausfuhrliche Beschreibung neuer Bakterien-Species. Zentralbl. Bakteriol. Parasitenk. Infekionskr. Hyg. Abt. 2. 114:520--537.

114. Nikitin, D. I., and L. V. Vasilyeva. 1967. A new soil organism Agrobacterium polyspheroidum n.sp. Izv. Akad. Nauk. SSSR, Ser. Biol. 3:443-444 (in Russian, English summary).

115. Omelianski, W. 1903. Uber die Zersetzung der Ameisensäure durch Mikroben. Zentralbl. Bakteriol. Parasitenk. Infekionskr. Hyg. Abt. 2. 11:117-189.

116. Pacheco, G. 1933. A posicão sistematica das bacterias das febres ondulantes. Rev. Soc. Paul. Med. Vet. 3:1-14.

117. Pagliani, Maggiora, and Fratini. 1887. Esame dei terreni colle colture; forme e caraterri dei microorganismi osservati. G. Soc. Ital. Ig. IX:584593.

118. Patrick, R., and C. H. Werkman. 1933. Bacteria fermenting xylan. Iowa State Coll. J. Sci. 7:407-418.

119. Peshkov, M. A. 1937. The variability, life-cycle and nuclear forms of a saprophytic water microbeAchromobacter epsteinii mihi. Biol. Zh. 6:9711006.

120. Piéchaud, D., M. Piéchaud, and L. Second. 195I. Etude de 26 souches de Moraxella lwoffi. Ann. Inst. Pasteur (Paris) 80:97-99.

121. Pohl, F. 1892. Ueber Kultur und Eigenschaften einiger Sumpfwasser-Bacillen und über die Anwendung alkalischer Nahrgelatine. Zentralb. Bakteriol. Parasitenk. Infektionskr. Hyg. Abt. 1, 11: $141-145$

122. Prévot, A. R. 1961. Traité de Systématique Bactérienne, p. 1-711, vol. 2. Dunod, Paris.

123. Poinar, G. O., and G. M. Thomas. 1965. A new bacterium, Achromobacter nematophilus sp. nov. (Achromobacteriaceae: Eubacteriales) associated with a nematode. Int. Bull. Bacteriol. Nomencl. 
Taxon. 15:249-252.

124. Ravenel, M. P. 1896. Notes on the bacteriological examination of the soil of Philadelphia. Mem. Nat. Acad. Sci. 8:3-41.

125. Rubentschik, L. 1925. Über die Lebenstätigkeit der Urobacterien bei einer Temperature unter $\mathrm{O}^{\circ}$. Zentralbl. Bakteriol. Parasitenk. Infektionskr. Hyg. Abt. 2. 64:166-174.

126. Rubentschik, L. 1926. Über einige neue Urobakterienarten. Zentralbl. Bakteriol. Parasitenk. Infektionskr. Hyg. Abt. 2. 66:161-180.

127. Rubentschik, L. I., and D. G. Goicherman. 1935. On microbiology of mud lakes. 1. Investigation of Kuyal'nitskiy liman. Mikrobiologiya 4:403-420 (in Russian)

128. Russakova, G. S. and V. S. Butkevich 1941. Denitrification without making use of nitrates as a source of nitrogen. Mikrobiologiya 10:137-162 (in Russian)

129. Russell, H. L. 1892. Untersuchungen uber im Golf von Neapel lebende Bacterien. Z. Hyg. Infektionskr. 11:165-206.

130. Ruiz-Hertera, J. 1970. Achromobacter starkeyi n.sp. a methionine decomposing bacterium isolated from the soil. Antonie van Leeuwenhoek J. Microbiol. Serol. 36:329-333

131. Samuels, S. B., B. Pittman, H. W. Tatum, and W. B. Cherry. 1972. Report on a study set of Moraxella and allied bacteria. Int. J. Syst. Bacteriol. 22:1938 .

132. Schaub, I., and F. Hauber. 1948. A biochemical and serological study of a group of identical unidentifiable gram-negative bacilli from human sources. J. Bacteriol. 56:379-390.

133. Severi, R. 1946. La patogenicitá degli Achromobatteri per l'uomo. Prima osservazione sull'azione patogena di questi germi. Epididimite cronica da "Achromobacter spermophilum" n.sp. Boll. Ist. Sieroter. Milan 25:224- 230.

134. Shieh, H. S. 1964. Aerobic degradation of choline. 1. Fermentation of choline by a marine bacterium, Achromobacter cholinophagum n.sp. Can. J. Microbiol. 10:837-842.

135. Shimwell, J. L. 1937. Study of a new type of beer disease bacterium (Achromobacter anaerobium sp. nov.) producing alcoholic fermentation of glucose. J. Inst. Brew. 43:507-509.

136. Smart, H. F. 1932. A new bacterial species isolated from strawberries, Proc. 33rd Annu. Meet. Soc. Amer. Bacteriol., Baltimore. J. Bacteriol. 23:41 42.

137. Snieszko, S. F. 1957. Genus IV. Aeromonas Kluyer and van Niel 1936, p. 189-193. In R. S. Breed, E. G. D. Murray and N. R. Smith (ed.), Bergey's manual of determinative bacteriology, 7th ed., The Williams \& Wilkins Co., Baltimore.

138. Sonnabend, W. 1963. Eine neue AlcaligenesArt Alcaligenes giganteus n.sp. Arch. Hyg. 147:609-615.

139. Spalla, C., G. Biffi, and E. Rocca. 1961. Esigenza per vitamin $\mathbf{B}_{12}$ citosina desossiriboside $\mathrm{E}$ citosina desossiribotide in Achromobacter terrestris n.sp. G. Microbiol. 9:130-134.

140. Steinhaus, E. A. 1941. A study of bacteria associ- ated with thirty species of insects. J. Bacteriol. 42:757-790.

141. Sreenivasan, A. 1956. New species of marine bacteria tolerating high concentrations of copper. Curr. Sci. 25:92-93

142. Stanier, R. Y. 1941. Studies on marine agar-digesting bacteria. J. Bacteriol 42:527-558.

143. Stanier, R. Y., N. J. Palleroni, and M. Doudoroff. 1966. The aerobic pseudomonads: a taxonomic study. J. Gen. Microbiol. 43:159-271.

144. Stenzel, W., and W. Mannheim. 1963. On the classification and nomenclature of some non motile and coccoid diplobacteria exhibitng the properties of Achromobacteriaceae. Int. Bull. Bacteriol. Nomencl. Taxon. 13:195-200.

145. Stutzer, M. J., and W. J. Wsorow. 1927. Uber Infektionen der Raupen der Wintersautenle (Euxoa segetum Schiff). Zentralbl. Bakteriol. Parasitenk. Infektionskr. Hyg. Abt. 2. 71: 113-129.

146. Szybalski, W. 1950. A comparative study of bacteria causing mustiness in eggs. Nature (London) 165:733-734.

147. Takeda, S. 1936. On the relation between the salmon hatching and the bacterial content of the feeding water. Zentralbl. Bakteriol. Parasitenk. Infektionskr. Hyg. Abt. 2. 94:33-64

148. Thornley, M. J. 1967. A taxonomic study of Acinetobacter and related genera. J. Gen. Microbiol. 49:211-257.

149. Tulecke, W., S. W. Orenski, R. Taggart, and L. Colavito. 1965. Isolation of an organism resembling Achromobacter liquefaciens. J. Bacteriol. 89:905-909.

150. Turner, A. W. 1927. Achromobacter perolens (n.sp) the cause of mustiness in eggs. Aust. J. Exp. Biol. Med. Sci. 4:57-60.

151. Turner, A. W. 1954. Bacterial oxidation of arsenite. I. Description of bacteria isolated from arsenical cattle-dipping fluids. Aust. J. Biol. Sci. 7:452-478.

152. Vaughan, V. C. 1892. A bacteriological study of drinking water. Amer. J. Med. Sci. 104:167-198.

153. von Lingelsheim, W. 1906. Die bakteriologischen Arbeiten der Kgl. Hygienischen Station zu Beuthen O. Schl. während der Genickstrarrepidemie in Oberschlesien in Winter 1904/05. Klin. Jb. 15:373-488.

154. Weinzirl, J. 1919. The bacteriology of canned foods. J. Med. Res. 39:349-413.

155. Weldin, J. C. 1927. The colon-typhoid group of bacteria and related forms. Relationships and classification. Iowa State Coll. J. Sci. 1:121-197.

156. Wells, N. A., and C. E. ZoBell. 1934. Achromobacter ichthyodermis, n.sp. the etiological agent of an infectious dermatitis of certain marine fishes. Proc. Nat. Acad. Sci. U.S.A. 20:123-126.

157. White, G. F. 1921. The cause of European foul brood. U.S. Dep. Agr. Bur. Entomol. 157:1-15.

158. Wiidik, R. W. 1950. Weissfäule von Hühnereirenbedingt durch eine AchromobakterartAchromobacter ammoniagenes (n.sp.) Nord. Vet. Med. 2:789-803.

159. Wright, J. H. 1895. Report on the results of an examination of the water supply of Philadelphia. 
Mem. Nat. Acad. Sci. 7:422-484.

160. Zimmermann, O. E. R. 1890. Die Bakterien unserer Trink and Nutzwässer, insbesondere des Wassers der Chemnitzer Wasserleitung. I. Reihe. In Elfter Berichte naturwiss Ges. Chemnitz 54154.

161. ZoBell, C. E. 1943. The effect of solid surfaces upon bacterial activity. J. Bacteriol. 46:39-56.

162. ZoBell, C. E., and E. C. Allen. 1935. The significance of marine bacteria in the fouling of submerged surfaces. J. Bacteriol. 29:239-251.

163. ZoBell, C. E., and H. C. Upham. 1944. A list of marine bacteria including descriptions of sixty new species. Bull. Scripps. Inst. Oceanogr. 5:239-292. 